\title{
A utilização da laserterapia na prevenção e tratamento da mucosite bucal: uma
}

\section{revisão da literatura}

The use of laser therapy in the prevention and treatment of oral mucositis: a literature review

El uso de la terapia con láser en la prevención y tratamiento de la mucositis oral: una revisión de la literatura

Recebido: 11/11/2020 | Revisado: 13/11/2020 | Aceito: 29/12/2020 | Publicado: 03/01/2021

Flávio Murilo Lemos Gondim ORCID: https:orcid.org/0000-0002-2327-9986 UNIESP Centro Universitário, Brasil E-mail: flaviomurillo@hotmail.com

Breno Estevam Silva de Souza ORCID: https://orcid.org/0000-0001-5590-7441 UNIESP Centro Universitário, Brasil E-mail: brenno.estevam@gmail.com

\begin{abstract}
Resumo
Este trabalho é uma revisão de literatura, realizado entre junho 2019 e junho de 2020, que se dedicou a analisar artigos publicados na última década, sobre a utilização da laserterapia em pacientes acometidos por mucosite bucal, buscando relatar os fundamentos científicos que justifiquem a utilização desta medida terapêutica e as melhorias na qualidade de vida dos pacientes que se submeteram ao tratamento. Método: Busca realizada nas bases de dados Pubmed®, Scielo, Medline e LILACS, sendo descartados os artigos que não eram disponibilizados na íntegra ou cuja abordagem se distanciava do tema proposto. Conclusão: Os autores analisados defendem que houve uma considerável melhoria na qualidade de vida dos pacientes acometidos por mucosite bucal, quando submetidos a laseterapia de baixa intensidade, principalmente diante do controle da dor e dos processos inflamatórios, bem como, no reestabelecimento das funções bucais. Reforçam ainda a necessidade de capacitação dos profissionais que utilizam a laserterapia e a inclusão dos mesmos na equipe multidisciplinar.
\end{abstract}

Palavras-chave: Unidade hospitalar de odontologia; Estomatite; Terapia com luz de baixa intensidade.

\begin{abstract}
This study is a literature review, carried out between June 2019 and June 2020, which was dedicated to analyzing articles published in the last decade, on the use of lasotherapy in patients affected by oral mucositis, seeking to report the scientific foundations that justify the use of this therapeutic measure and improvements in the quality of life of submitted patients who underwent treatment. Method: Search carried out in the Pubmed®, Scielo, Medline and LILACS databases, discarding articles that were not available in full or whose approach was distant from the proposed theme. Conclusion: The analyzed authors argue that there was a considerable improvement in the quality of life of patients affected by oral mucositis, when submitted to lasetherapy, mainly in view of the control of pain and inflammation, as well as, in the restoration of oral functions. They also reinforce the need for training professionals who use laser therapy and their inclusion in the multidisciplinary team.
\end{abstract}

Keywords: Dental service, Hospital; Stomatitis; Low-level Light therapy.

\section{Resumen}

Este estudio es una revisión de la literatura, realizada entre junio de 2019 y junio de 2020, que se dedicó al análisis de artículos publicados en la última década, sobre el uso de lasetherapy en pacientes afectados por mucositis oral, buscando informar los fundamentos científicos que justifican el uso de esta medida terapéutica y mejoras en la calidad de vida de los pacientes sometidos a tratamiento. Método: Búsqueda realizada en las bases de datos Pubmed®, Scielo, Medline y LILACS, descartando artículos que no estaban disponibles en su totalidad o cuyo enfoque estaba alejado del tema propuesto. Conclusión: Los autores analizados argumentan que hubo una mejora considerable en la calidad de vida de los pacientes afectados por mucositis oral, cuando se los sometió a la última terapia, principalmente en vista del control del dolor y la inflamación, así como en la restauración de las funciones orales. También refuerzan la necesidad de capacitar a profesionales que usan la terapia con láser y su inclusión en el equipo multidisciplinario. Palabras clave: equipo de odontología hospitalaria; Estomatite, Terapia por luz de baja itensidade. 


\section{Introdução}

O estudo de Andrade, Clark \&Ferreira (2014), apontou que a utilização do laser como opção terapêutica é visualizada na área da biomedicina desde a década de 60, através de Theodore Maiman, tendo um dos estudos pioneiros publicados sobre os efeitos do laser de baixa potência em 1983, através da irradiação de laser HeNe (Hélio-Neônio), sobre lesões de camundongos durante 14 dias ininterruptos.

Miguel, et al., (2017), definiu que a palavra Laser, na verdade é uma sigla inglesa que significa de "Light Amplification by the Stimulated Emission of Radiation”, e a utilização em odontologia tem passado por uma ininterrupta evolução, desde o início do século XX, sendo cada vez mais aplicado nas diversas especialidades odontológicas, em procedimentos diagnósticos ou terapêuticos, sendo necessário distingui-lo em dois tipos: O laser de alta potência ou cirúrgico e o laser de baixa potência ou terapêutico.

Gomes, et al., (2013), em seu estudo descritivo, reforçou a relevância que o Cirurgião-Dentista (CD) exerce ao buscar novas técnicas que ofereçam maior conforto ao paciente, tentando transformar a Odontologia curativa, dolorosa e traumática em uma que utiliza métodos preventivos, aliada a equipamentos avançados e materiais modernos que proporcionam tratamentos rápidos, conservadores e mais confortáveis para os pacientes.

E reforçou que a odontologia tende a incorporar alternativas menos invasivas, em vias de diminuir a dor e o desconforto na constância e posteriormente aos procedimentos odontológicos. Desta forma, a terapia a laser de baixa intensidade (TLBI) se coloca como uma opção de terapia viável, na medida em alcança benefícios para a área irradiada, como ativação da microcirculação, produção de novos capilares, efeitos anti-inflamatórios e analgésicos, além de estímulo ao crescimento e a regeneração celular (Gomes, et al., 2013).

Neste sentido, o supracitado autor, relatou os efeitos advindos da terapia com laser de baixa intensidade como sendo um procedimento que pode levar a uma aceleração no processo de cicatrização, o que se atribui a variados processos metabólicos, através da conversão da energia luminosa, que se transforma, por meio de processos bioquímicos e fotofísicos, em energia útil para a célula.

Siqueira, et al., (2015), trouxe à lume que os lasers de baixa potência (LBPs), possuem diversas indicações, entre elas, diagnóstico de cárie, utilizando um laser que identifica diferenças de comportamento óptico entre o tecido sadio e o cariado, estimular a formação de dentina reparadora, reduzir a sensibilidade dentinária e a dor nos casos de hiperemia, auxiliar a anestesia nos casos de pulpite severa, promover maior velocidade de reparação do osso na região periapical, diminuição do sangramento e edema nos casos de gengivite e periodontite e diminuir a sintomatologia dolorosa na síndrome da ardência bucal, alveolite, pericoronarite, além do tratamento da mucosite oral.

Rozza, et al., (2011), relatou que o termo "mucosite bucal" surgiu na década de 1980, sendo descrito como uma inflamação na mucosa decorrente da toxicidade causada pelos quimioterápicos, que não distinguem as células tumorais, que se replicam rapidamente, das normais, o que causa injuria nas células da mucosa bucal, tornando o tecido atrófico, repercutindo no rompimento do tecido epitelial.

Miguel, et al, (2017), corroborou com o autor retro no sentido de que a mucosite é uma complicação recorrente entre os pacientes submetidos a quimioterapia e radioterapia, e acrescentou que se trata de uma patologia que agride o trato gastrointestinal e faringe, não apenas a mucosa bucal. Inicialmente a mucosite se apresenta de forma assintomática, posteriormente fica evidenciado o eritema, a sensação de ardência e o desconforto ao se alimentar, o que repercute na qualidade de vida do paciente.

A avaliação da MO pode ser realizada por critérios de toxicidade estabelecidos pelo National Cancer Institute (NCI), Conforme estudo de Figueiredo, et al., (2013), este método estabelece grau 0 na ausência de MO; grau 1 quando existe úlceras indolores, eritema ou dor leve na ausência de úlceras; grau 2 na presença de eritema doloroso, edema ou úlceras, mas 
alimentar-se ou deglutir é possível; grau 3 na presença de eritema doloroso, edema, ou úlceras com necessidade de nutrição parenteral; grau 4 quando há ulceração grave ou necessidade de nutrição parenteral ou intubação profilática; e grau 5 em caso de morte relacionada à toxicidade.

A literatura aponta, segundo Campos, et al., (2013), que a maioria das células respondem à irradiação com a luz monocromática originada dos lasers e LEDs, alterando seu metabolismo. A mitocôndria é, geralmente a localização inicial da ação da luz, sendo o citocromo $\mathrm{C}$ oxidase o principal cromóforo. Este é o principal fato para as mudanças posteriores no metabolismo celular, incluindo alterações na via do ATP, a qual se comporta como fonte de energia e sinalização crítica para comunicação celular e tecidual, além de ter relação com a proliferação celular e alívio da dor.

Andrade, et al., (2020), apontou que a terapia antineoplásica em região de cabeça e pescoço é dividida em três modalidades que podem ser associadas ou não, a saber, radioterapia, quimioterapia e cirurgia, a depender do estágio e região da doença, entretanto, tecidos sadios que se localizam no perímetro ou próximo aos campos irradiados terminam sendo atingidos, desencadeando efeitos colaterais que podem se manifestar ao tempo ou em seguida a radioterapia, se estendendo por toda vida.

Em seu estudo, Reolon, et al., (2017), destacou que o mecanismo estabelecido pela mucosite por radiação seria semelhante ao da mucosite por quimioterapia, embora dependa de múltiplos fatores como tipo de radiação, volume do tecido irradiado, doses, esquema de fracionamento, e ainda fatores relacionados ao paciente como idade, hábitos e condição clínica.

Entre os pacientes acometidos por tumores de cabeça e pescoço que se submeteram a radioterapia ou quimioterapia, Florentino, et al., (2015), relatou que 90 à 97\% apresentam mucosite oral na constância do tratamento antineoplástico, o que justifica o acompanhamento odontológico hospitalar. A radioterapia na região de cabeça e pescoço exacerba o efeito da mucosite oral, considerando o efeito ionizante e deletérios sobre o DNA, que causa injuria as mucosas a partir de uma determinada dose, e quando este tratamento é associado a quimioterapia as chances do paciente desenvolver a mucosite oral é ainda maior.

Várias abordagens terapêuticas têm sido empregadas para o tratamento da mucosite oral, o trabalho de Santos, et al., (2019), citou entre elas, a crioterapia oral, fatores de crescimento, antinflamatórios não esteroidais, analgésicos opióides e a laserterapia.

Diante da contextualização realizada acima, surge a seguinte problemática: a laserterapia se apresenta como uma alternativa viável no tratamento da mucosite oral? Em vias de responder o questionamento retro, foi traçado como objetivo, revisar a literatura contemporânea na intenção de detectar as contribuições e resultados dessa opção terapêutica no tratamento da Mucosite Oral

\section{Metodologia}

O percurso metodológico do presente estudo se estabeleceu através de uma revisão bibliográfica, de natureza qualitativa descritiva exploratória, utilizando artigos publicados na última década sobre o tema em tela. Em vias de alcançar os objetivos traçados, construiu-se um resumo das investigações relacionadas com uma estratégia de intervenção específica através da aplicação de critérios explícitos e metodologia de busca, análise crítica e condensação de informações selecionadas, apontando para os resultados alcançados para preencher lacunas no conhecimento, identificando áreas que necessitam de pesquisas futuras. Foi realizada uma busca na base de dados Pubmed®, Scielo, Medline e LILACS, utilizando os descritores na língua portuguêsa: Unidade Hospitalar de Odontologia; Estomatite; Terapia com Luz de Baixa Intensidadade, e na língua inglesa: Dental Service, Hospital; Stomatitis; Low-Level Light Therapy. Para delimitar o estudo, foram estabelecidos como critérios de inclusão: artigos escritos em português, inglês e espanhol, disponíveis na íntegra e 
excluídos aqueles que se distanciavam do tema proposto, como por exemplo, aqueles que tratavam exclusivamente de laser de alta potência ou cirúrgico, sendo excluídos também os artigos que não eram de livre acesso.

A pesquisa se deu entre os meses de junho de 2019 e junho de 2020, sendo realizada em um primeiro momento uma busca nas bases de dados Scielo, PubMed, Medline e LILACS, sendo selecionadas 67 publicações. Após leitura minuciosa, utilizando os critérios de inclusão e exclusão, 16 publicações foram julgadas adequadas a integrar a seleção desta revisão, sendo 8 artigos de revisão de literatura, 4 estudos descritivos, 1 estudo prospectivo randomizado, 1 metanálise, 1 resolução e 1 estudo quantitativo.

\section{Resultados e Discussão}

O Conselho Federal de Odontologia, através da Resolução CFO nº 82/2008, estabeleceu a laserterapia como integrante as práticas integrativas e complementares em saúde bucal, e deu outras providências, tratando exclusivamente do tema em no Capítulo VI, nesse sentido vejamos o que diz a respectiva normativa:

\section{CAPÍTULO VI}

\section{DA LASERTERAPIA}

Art. 31. A Laserterapia em Odontologia tem como objetivo capacitar os Cirurgiões-Dentistas de maneira a assegurar a prática profissional de forma ampla e segura. Respeitando o limite de atuação do campo profissional do CirurgiãoDentista.

Art. 32. São atribuições do habilitado em Laserterapia em Odontologia:

I - aplicar a interação de luz com os tecidos biológicos (terapia fotodinâmica); e,

II - aplicações clínicas dos lasers em alta e baixa intensidade e LEDs nas diversas áreas da Odontologia.

Art. 33. O Cirurgião-dentista, que na data de publicação desta Resolução, comprovar vir utilizando Laserterapia, há cinco anos dentro dos últimos dez anos, poderá requerer habilitação, juntando a documentação para a devida análise pelo Conselho Federal de Odontologia.

Art. 34. Também poderá ser habilitado o Cirurgião-Dentista aprovado em concurso que deverá abranger provas de títulos, escrita e prática-oral, perante Comissão Examinadora a ser designada pelo Conselho Federal de Odontologia. Parágrafo único. Para se habilitar ao disposto nos artigos 33 e 34, o interessado deverá apresentar requerimento ao Conselho Regional onde tenha inscrição principal até 180 (cento e oitenta) dias após a publicação desta Resolução, acompanhado da documentação pertinente.

Art. 35. Também será habilitado o Cirurgião-Dentista que apresentar certificado de curso portaria do pelo Conselho Federal de Odontologia, que atenda às seguintes disposições:

I - que o certificado seja emitido por:

a) instituições de ensino superior;

b) entidades especialmente credenciadas junto ao MEC e/ou CFO; e, c) entidades de classe, sociedades e entidades de Laserterapia, devidamente registrada no CFO.

II - Que a carga horária mínima do curso seja de 60 horas entre teórica e prática;

III - que o curso seja coordenado por Cirurgião-Dentista habilitado em Laserterapia pelo Conselho Federal de Odontologia; e,

IV - que o corpo docente seja composto por cirurgiões dentistas habilitados na prática de Laserterapia e profissionais da área da saúde com comprovado conhecimento técnico científico.

Art. 36. Do conteúdo programático mínimo deverão constar conhecimentos que atendam aos seguintes tópicos:

a) aspectos físicos e biológicos do uso de laser em Odontologia;

b) uso clínico dos diferentes cumprimentos de onda, incluindo os lasers de alta e baixa potência;

O termo LASER, que significa Light Amplification by Stimulated Emission of Radiation (Amplificação da luz por emissão estimulada de radiação, em português) consiste de uma radiação eletromagnética, unidirecional, monocromática, com feixe estreito, propagação paralela (colimação) e com as ondas dos fótons em fase (coerência). (GOMES \& SCHAPOCHNI, 
Magnabosco Neto \& Westphalen (2013), afirmou o Laser se trata de radiação eletromagnética não ionizante, extremamente concentrada, que possui capacidade de emitir luz com comprimento de onda único e definitivo, com características distintas.

Quando da absorção por diferentes tecidos, dependendo do laser, a radiação resulta em efeitos fotoquímicos, térmicos e não lineares. Essa interação com o tecido está diretamente relacionada com o comprimento de onda, dose ou fluência (densidade de energia), intensidade (densidade de potência), regime de operação do laser, taxa de repetição ou frequência do pulso e características óticas do tecido, como o coeficiente de absorção e espalhamento, para que se possa estabelecer o número de aplicações e dose ótima de radiação. (Neto, et al., 2013).

Em seu estudo, Rozza, et al., (2011), relata que os autores revisados sugerem que a diminuição dos streptococos mutans no período de quimioterapia pode possibilitar à instalação de micro-organismos patogênicos como Pseudomonas aeruginosa, cuja interação com a mucosa bucal é maior, podendo se relacionar com a MO.

A autora acima relatada, compartilha que além do adequado equilíbrio entre o ambiente bucal e a microbiota, a TLBI possibilita o controle de mediadores inflamatórios e acelera os mecanismos de reparo por meio de células mesenquimais e substâncias da matriz extracelular, demonstrando resultados eficientes na prevenção das mucosites. (Rozza, et al., 2011).

O tratamento antineoplásico, abrangendo a radioterapia e/ou quimioterapia, possui capacidade de induzir a danos celulares no epitélio, na mucosa oral e nas estruturas glandulares salivares, prejudicando as suas funções e, consequentemente, promovendo alterações quantitativas e qualitativas, que se manifestam, comumente, como mucosite oral - a principal complicação estomatológica em pacientes com câncer. (Reolon, et al., 2017)

Baseado nisso, o estudo em questão averiguou a qualidade de vida dos pacientes com mucosite oral induzida pelos tratamentos antineoplásicos, visando à importância de suprir e assistir à população oncológica perante os impactos na qualidade de vida, assim como as limitações em razão da doença e a interferência do tratamento na vida social, para que seja restabelecida a máxima dignidade de vida possível. (Reolon, et al., 2017).

A Laserterapia de baixa intensidade proporciona alívio das dores agudas e crônicas, promovendo a analgesia imediata e temporária; podendo ainda ser incorporada no tratamento de mucosite oral, herpes, aftas, candidíase, nevralgias, xerostomia, paralisias faciais, dores articulares, inflamações e lesões na mucosa oral, hipersensibilidade dentinária, disfunção temporomandibular, problemas periodontais, tratamentos restauradores, ortodônticos e endodônticos, pós-operatórios cirúrgicos, cefaleias e outros. (Reolon, et al., 2017).

Campos, et al., (2013) verificou em seu estudo de caso, que a paciente relatou melhora no seu sintoma de boca seca, a xerostomia e informou que, trabalhos recentes têm demonstrado que a laserterapia pode ser auxiliar em casos de hipofunção das glândulas salivares com melhora da sintomatologia, ou seja mais uma indicação para o procedimento em comento.

Ainda há muito que se discutir a respeito das terapias complementares, como a TLBI, mas já é um avanço conseguir regulamentá-las. Hoje, sabe-se que os profissionais habilitados estão mais conscientes da importância do estudo para que melhores resultados sejam obtidos. (Gomes, et al., 2013).

Os cursos de habilitação em Laserterapia, de acordo com Gomes, et al., (2013), possuem a função garantir a devida prática, para que os profissionais não venham a exerce-las sem o conhecimento preciso, atingindo resultados indevidos e colocando em os pacientes, ou seja, tem por objetivo torná-los competentes. É oportuno, a definição de competência, considerando-a como a condição de agir eficientemente em uma situação, com amparo em conhecimentos, mas sem restringilos.

O laser de baixa potência, tratado por Miguel et al., (2017), também chamado de nomes diferentes tais como laser frio o laser terapêutico, cuja potência não ultrapassa os $0,5 \mathrm{~W}$, se apresenta entre a luz vermelha visível e o infravermelho (IR), dentro do espectro eletromagnético, desde os $630 \mathrm{~nm}$ até os $980 \mathrm{~nm}$. Na odontologia os lasers mais empregados são os de diodo 
que têm como meio ativo o Arsênio de Gálio e Alumínio, que emitem entre 808-830nm, com potência entre os 0,02 e 0,1W. Também se aplicam os lasers de Fosforo, Alumínio, Indio e Galio, com longitude de onda entre 635-690nm e potências entre 0,1 e $0,25 \mathrm{~W}$, bem como como o laser de He-Ne.

A utilização de laser de baixa intensidade na mucosa oral, conforme Figueiredo, et al., (2013), tem capacidade de prevenir a ocorrência de $\mathrm{MO}$ grau $\geq 3 \mathrm{em}$ pacientes submetidos à oncoterapia, neste sentido, os indivíduos submetidos à LT, esta profilaxia é cerca de nove vezes mais eficiente que a ausência de LT.

Para Andrade, et al., (2020), A radioterapia é, seguramente, uma eficaz opção terapêutica para as neoplasias malignas na região de cabeça e pescoço, entretanto, mesmo com toda tecnologia disponível, a terapia desencadeia efeitos colaterais indesejados que comumente afetam o ambiente bucal, o que exige do profissional preparo para o estabelecimento de estratégias preventivas e terapêuticas adequadas, garantindo melhoria na qualidade de vida que envolvem aspectos físicos e psicológicos.

Todos os autores revisados por Florentino, et al., (2015), concordam que o acompanhamento odontológico pós quimioterapia e radioterapia são relevantes na prevenção e cuidados da mucosite oral, além disso, há consenso entre os autores de que para a laserterapia obter resultados de analgesia e estimular a cicatrização deve ser realizada pelo menos três vezes por semana1, resguardando a proteção ocular por meio dos óculos.

O autor supracitado, relata também em seu estudo que a aplicação da laserterapia pode ser realizada usando a técnica da varredura ou da aplicação pontual, e ressalta que além dos benefícios clínicos já tratados, esta terapia não invasiva e de curso baixo diminui o gasto com analgésicos, além de outras despesas associadas aos efeitos deletérios das terapias antineoplásicas o que vem a servir de para adesão de novas políticas em saúde.

Santos, et al., (2019), propôs o protocolo, a seguir descrito, para o tratamento da MO: comprimento de onda de $633 \mathrm{~nm}$ a $685 \mathrm{~nm}$ ou $780 \mathrm{~nm}$ a $830 \mathrm{~nm}$, potência de $0,01 \mathrm{~W}$ a $0,15 \mathrm{~W}$, densidade de energia 2 a $3 \mathrm{~J} / \mathrm{cm} 2$ e não mais que $6 \mathrm{~J} / \mathrm{cm} 2 \mathrm{na}$ superfície tratada, no período de três vezes por semana até a melhora do quadro, em caso de MO grave, é possível realizar a laserterapia diariamente.

A MO é uma das patologias mais relacionadas a dor bucal, segundo Carvalho, (2015), podendo atingir um patamar que implica na incapacidade de nutrição, piorando a qualidade de vida e, até causando a modificação ou interrupção do tratamento, o que se correlaciona ao alto risco para a proliferação das células tumorais, reduzindo o controle loco regional do tumor. Ademais, existem evidências que um pequeno lapso temporal de paralisação da RT gera consequências negativas, estimando-se que para cada dia de RT interrompida, diminui $1 \%$ a taxa de controle local do tumor.

Em seu estudo, Andrade, Clark \& Ferreira (2014), relataram que a laserterapia, tem sido empregada objetivando promover melhora processos inflamatórios, analgesia, diminuição de edema, assim como, preservar tecidos e nervos adjacentes ao local da injúria. Esse resultado pode ser atingindo através de comprimentos de onda entre 600 e $1000 \mathrm{~nm}$ e potências de $1 \mathrm{~mW}$ a $5 \mathrm{~W} / \mathrm{cm} 2$. Os autores pontuam ainda, que potências muito baixas $(2,5 \mathrm{~W} / \mathrm{cm} 2)$ ou muito elevadas $(25 \mathrm{~W} / \mathrm{cm} 2)$, podem ocasionar efeitos contrários.

A LTBI, segundo Aquino, et al., (2020), é mais acessíveis devido seu custo e benefício, sendo opção terapêutica segura, não farmacológica, que melhora os tratamentos metabólicos mediante o processo de incorporação e fatores energéticos realizados pelos cromóforos, dos quais ocasionam modificações no funcionamento das mitocôndrias, e consequentemente, mudança respiratória da célula, com maior produção de adenosina trifosfato (ATP), gerando a ativação de espécies reagentes de oxigênio intracelular, resultando na multiplicação de fibroblastos, cadeias de colágenos e melhora nas respostas de eventuais inflamações resultando na melhor reparação do tecido. 


\section{Considerações Finais}

$\mathrm{Na}$ presente revisão de literatura, verificou-se que os autores analisados defendem que houve uma considerável melhoria na qualidade de vida dos pacientes acometidos por mucosite bucal, quando submetidos a laseterapia de baixa intensidade, principalmente diante do controle da dor, dos feitos da inflamação, regressão do edema, mas também no reestabelecimento das funções bucais.

Os resultados encontrados são variáveis, porém a utilização do laser de baixa intensidade foi considerada uma medida eficaz na prevenção e tratamento da mucosite oral, se apresentando como uma medida terapêutica eficaz diante de seu baixo custo, e alcance dos resultados desejados em curto prazo de tempo.

Alguns dos autores analisados, reforçam a necessidade de capacitação dos Cirurgiões-Dentistas para utilização do laser, e inserção dos mesmos na equipe multidisciplinar, principalmente, no que tange ao tratamento oncológico.

Os resultados encontrados apresentam semelhança, apesar de protocolos variados, entretanto, a maioria dos autores revisados concordam que novos estudos devem ser realizados afim de estreitar a relação entre a laserterapia de baixa intensidade e o tratamento de processos patológicos, preenchendo as lacunas ainda existentes

\section{Referências}

Andrade, F. S. S. D.; Clark, R. M. O.; Ferreira, M. L. (2014). Efeitos da laserterapia de baixa potência na cicatrização de feridas cutâneas Revista do Colégio Brasileiro de Cirurgiões. Rio de Janeiro. 41(2), 129-133.

Andrade, N. S., et al. (2020). Manejo multimodal para reabilitação oral de paciente irradiado em cabeça e pescoço: relato de caso. Research, Society and Development, 9(7), 1-12.

Berber, M. A. R., et al. (2017). Usos del láser terapéutico en Odontopediatría: Revisión de la literatura. Reporte de casos. ODOVTOS-International Journal of Dental Sciences. San José. 20(3), 51-59.

Campos, L., et al. (2013). Laserterapia no tratamento da mucosite oral induzida por quimioterapia: relato de caso. Revista da Associação Paulista de Cirurgiões-Dentistas. São Paulo. 62(2), 102-106.

Carvalho, P. A. G. (2015). Avaliação da laserterapia na prevenção de mucosite oral relacionada à radioterapia: comparação entre os efeitos de diferentes protocolos de laser de baixa potência. (Doutorado em Ciências). Fundação Antônio Prudente. São Paulo. p. 8-9.

Conselho Federal De Odontologia. (2008). Resolução CFO 82/2008 Reconhece e regulamenta o uso pelo cirurgião-dentista de práticas integrativas e complementares à saúde bucal. Conselho Federal de Odontologia. 13-15.

Figueiredo, A. L. P., et al. (2013). Laser terapia no controle da mucosite oral: um estudo de metanálise. Revista da Assciação Médica Brasileira. São Paulo. 59(5), 467-474.

Florentino, A. C. A. et al. (2015). Tratamento da mucosite oral com laser de baixa potência: revisão sistemática de literatura. Revista de Ciências Médicas. Campinas. 24(2), 85-92.

Gomes, M. N. C. et al. (2013). O ensino da terapia a laser de baixa intensidade em Odontologia no Brasil. Revista da Faculdade de Odontologia da Universidade de Passo Fundo. Passo Fundo. 18(1), 32-36.

Gomes, C. F., Schapochnik, A. (2017). O uso terapêutico do LASER de Baixa Intensidade (LBI) em algumas patologias e sua relação com a atuação na Fonoaudiologia. Distúrb Comum. São Paulo. 29(3),. 570-578.

Koche, J. C. (2011). Fundamentos de metodologia científica. Petrópolis: Vozes.

Ludke, M. \& Andre, M. E. D. A. (2013). Pesquisas em educação: uma abordagem qualitativa. São Paulo: E.P.U. E.

Magnabosco Neto, A., Westphalen, F. H. (2013). Efetividade profilática e terapêutica do laser de baixa intensidade na mucosite bucal em pacientes submetidos ao tratamento do câncer. Revista da Faculdade de Odontologia da Universidade de Passo Fundo. Passo Fundo. 16(2), $246-253$.

Pereira, A. S. et al (2018). Metodologia da pesquisa científica. [free ebook]. Santa Maria: UAB/NTE/UFSM.

Reolon, L. Z. et al. (2017). Impacto da laserterapia na qualidade de vida de pacientes oncológicos portadores de mucosite oral. Revista Odontológica da UNESP. Araraquara. 46(1), 19-27.

Santos, J. T. L.; Neto, J. A. F, Catão, M. H. C. V. (2019). Fototerapia no tratamento da mucosite oral: uma revisão de literatura. Arquivo em Odontologia. Belo Horizonte. 55, 1-10.

Rozza, R. E., Ferreira, S. G., Paulo, H. C. S. (2011). Aspectos clínicos e prevenção das mucosites bucais - revisão. Revista da Faculdade de Odontologia da Universidade de Passo Fundo. Passo Fundo. 16(2), 217-223. 
Research, Society and Development, v. 10, n. 1, e5910110149, 2021

(CC BY 4.0) | ISSN 2525-3409 | DOI: http://dx.doi.org/10.33448/rsd-v10i1.10149

Silva Neto, J. M. A. et al. (2020). Aplicação da laserterapia de baixa intensidade na odontologia: revisão integrativa. Revista Eletrônica Acervo Saúde.39, 110 .

Siqueira, M. B. L. D. et al. (2015). A terapia com laser em especialidades odontológicas. Revista Cubana de Estomatologia. Cuba. 59(2), 143-149. 